\title{
Haptic Feedback using Local Models of Interaction
}

\author{
Daniela Constantinescu \\ Department of Electrical and Computer Engineering \\ University of British Columbia \\ Vancouver, Canada \\ danielac@ece.ubc.ca
}

\author{
Septimiu E. Salcudean \\ Department of Electrical and Computer Engineering \\ University of British Columbia \\ Vancouver, Canada \\ tims@ece.ubc.ca
}

\author{
Elizabeth A. Croft \\ Department of Mechanical Engineering \\ University of British Columbia \\ Vancouver, Canada \\ ecroft@mech.ubc.ca
}

\begin{abstract}
A local model of interaction proposed for haptic rendering of rigid body motion is extended to the rigid body manipulation of articulated structures. In the proposed local model, the motion constraints imposed on the user by the articulated structure are rendered through the local dynamics, while those imposed by other virtual objects are rendered through impulsive, penalty, and friction contact forces. The proposed local model is used to interface a planar haptic device to a virtual world comprised of both rigid objects and articulated structures moving within an enclosure of rigid walls.
\end{abstract}

\section{Introduction}

By allowing users to touch and manipulate virtual environments, haptic devices are potentially useful computer interfaces for many applications, ranging from physical skill training to entertainment. Due to their prospective advantages, significant research effort has been dedicated to the development of haptic control [8], [2] and simulation algorithms [19], [6], [15] over the last ten years. Regardless of this effort, only a limited number of haptic aplications [3], [4] exist to date.

A major obstacle to the wide-spread use of haptic devices is the lack of a technique to allow the interfacing of a device to an arbitrary virtual environment simulation while guaranteeing stable interaction and providing realistic force feedback. Such a technique is challenging to develop because of the constraints imposed on the simulation by the device controller, by our sense touch, and, very often, by the application itself.

One constraint is the force "refresh" rate which is of the order of hundreds of $\mathrm{Hz}$. The device controller needs this high "haptic rate" in order to create an adequate perception of rigidity, while our sense of touch needs it in order to avoid perceptual artifacts, such as vibrations. Another constraint is the passivity of the numerical methods used to generate the virtual environment. The device controller requires a passive simulation in order to guarantee stable interaction while transferring power from the virtual environment to the user [6]. A further constraint is the need for realism of the simulated forces. A large number of haptic applications require realistic forces and are useful only if they accurately represent a physical phenomenon. Nevertheless, reported rendering frequencies of typical interactive physically-based simulations of multi rigid body virtual environments are only of the order of tens of $\mathrm{Hz}$ [18], and they can slow down further during complex interactions. In response to the need for interaction stability and the need for a high haptic rate, two general purpose approaches have been suggested.

One approach improves the real time performance of the virtual environment via new collision detection methods [11]. These methods allow general purpose rigid body dynamic algorithms to be used for rendering complex virtual environments. Simulations developed using this technique achieve required haptic rates only by using these collision detection algorithms in conjunction with non-passive numerical methods (penalty-based dynamic algorithms and fixed step forward integration routines). Thus, stability of interaction is not guaranteed [11].

Another approach decouples the force control loop from 
the virtual environment, thus eliminating the haptic rate demand from the simulation. As a result, passive virtual environments of increased complexity can be rendered. In this approach, a convincing haptic experience is created only if the forces supplied to the control loop during one simulation step are suitable approximations of the forces acting on the object manipulated by the user (called the virtual tool) in the virtual environment. Practically, this requires a local model of the interaction to be used by the control loop to calculate its setpoints [1], [5]. As the local model representation of the interactions between the virtual tool and the virtual environment is improved, so is the feedback provided to the user.

The present research proposes to take the second approach. That is, to meet the need for interaction stability and the need for a high haptic rate by decoupling the force control loop from the simulation through a local model of interaction. The proposed model can be used for adding realistic force feedback to the rigid body manipulation of both rigid objects and articulated structures. In this model, the interaction between the virtual tool and the virtual environment is approximated by limiting the interaction of the virtual tool to neighboring objects.

As shown in Figure 1, the local model consists of a dynamic proxy of the virtual tool and the geometric constraints representing the contacts between the virtual tool and neighboring objects. The dynamic proxy encapsulates the motion constraints imposed on the user by the articulated structure. The geometric constraints encapsulate the motion constraints imposed on the user by objects neighboring the virtual tool. As shown in Figure 1, the virtual environment coordinates the virtual tool and the proxy using proxy state information, while the controller coordinates the proxy and the device using both state and force information.

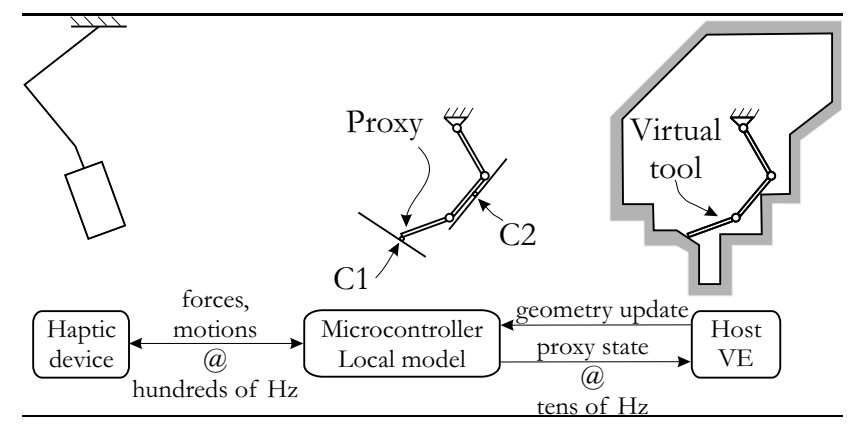

Figure 1. Communication between the virtual environment, the local model of interaction, and the haptic device.

The virtual environment maintains the quality of the local approximation by updating the local geometry at each step of the simulation. Local geometry improves the real- ism of the haptic feedback in two ways: (i) it allows much stiffer environments to be rendered because it simplifies local collision detection and, thus, it allows a high control rate to be achieved; and (ii) it allows users to feel physical phenomena requiring fast force transitions, such as collisions and stick-slip friction. Undesirable discontinuities that may arise in the local interaction forces due to geometry updates are avoided using the techniques discussed in [10].

The rest of the paper is organized as follows. Section 2 briefly overviews three areas of related research, namely: (i) local models used for decoupling of the force control loop from the simulation, (ii) haptic manipulation of articulated structures, and (iii) contact models used for haptic rendering of rigid contact. Section 3 describes the local model of interaction proposed in this work for the haptic manipulation of articulated structures and the local modeling of contacts. Section 5 presents the experimental setup used to implement the proposed local model and implementation results. Conclusions and future work are discussed in Section 6.

\section{Related research}

The earliest local model of force computation was developed for point interaction in a virtual environment and consisted of a geometric description of the virtual constraint [1]. Force discontinuities due to geometry updates were later reduced through the use of kinematic constraints [18] and then eliminated through linear interpolation between old and new constraints [13].

The first local model for rigid body interaction in a virtual environment included all active constraints, enabling users to feel crisp, stiff contacts [5]. However, the model exhibited instabilities during tightly constrained motions of the virtual tool (peg-in-hole tasks). Therefore, it was used to constrain only the translational degrees of freedom of the device and rotation was constrained through a virtual coupler.

The first local model for rigid body interaction in a virtual environment used to constrain all degrees of freedom of a device was proposed in [10]. It included all constraints within a neighborhood of interest of the virtual tool, thus displaying increased robustness to delay. Moreover, it included a dynamic proxy that allowed much higher contact stiffnesses to be achieved through the coordination of both forces and positions between the proxy and the haptic device. This local model is extended in the present work to the rigid body manipulation of articulated structures.

In prior research, haptic manipulation of articulated structures was performed through a proxy that played the role of a virtual coupler and enabled point interaction with linkages [14], [15]. In these works, the focus was on developing efficient algorithms for simulating complex structures at the high haptic rates. Therefore, the dynamics were de- 
scribed in joint space. Constraint-based collision response algorithms were also developed for the linkages. These algorithms are appropriate for a single collision at one time step. Moreover, the collision impulses are filtered through the virtual coupler before they are applied to the user.

Applying impulses to the user was first proposed in [16] with the aim of improving contact stability and the perception of rigidity. The impulses comprise forces acting over one haptic cycle when a contact is intiated. They increase the damping of the contact instantaneously, dissipating most of the kinetic energy of the haptic device.

In the context of rigid body interaction in a virtual environment, collision impulses were first used in a haptics application in [7]. In that implementation, the virtual environment was simulated using an impulse-based technique and difficulties arose due to multiple collisions occuring during one haptic step.

Collision impulses combined with penalty-based contact forces were used to render rigid body interaction in a planar virtual world in [9]. The present work is the first to use impulses in a local model of interaction. Furthermore, it develops a collision response model suitable for multiple collisions during one haptic step.

\section{Approach}

The proposed local model of interaction eliminates the force feedback loop between the haptic device and the virtual environment, thus shifting the communication delay between them from delay in force computation to delay in geometry update. Continuity of geometry at updates is maintained in this local model through prediction and interpolation of constraints [10], while the force feedback provided to the user is improved through the techniques presented in the following sections. These techniques allow the user to operate virtual linkages and to perceive collisions more realistically.

\subsection{Local dynamics}

Haptic manipulation of linkages is incorporated in the proposed local model by defining the virtual tool to be the entire articulated structure if the user holds one of its links (see Figure 1). This definition encapsulates motion constraints imposed on the user by the linkage topology into the local proxy dynamics and it requires neighboring contacts of all links to be sent to the local model.

Regardless of whether the virtual tool is a rigid object or a linkage, the local proxy dynamics can be described in configuration space through:

$$
\ddot{\boldsymbol{q}}=\boldsymbol{D}^{-1}(\boldsymbol{\tau}-\boldsymbol{G})
$$

where $\boldsymbol{q}, \dot{\boldsymbol{q}}$, and $\ddot{\boldsymbol{q}}$ are vectors of configuration space postions, velocities, and accelerations, $\boldsymbol{D}$ is the configuration space mass matrix, and $\boldsymbol{G}$ are gravitational forces. For computational simplicity, Coriolis and centrifugal effects have been neglected in Equation (1). $\tau$ is the vector of configuration space contact forces and is computed by:

$$
\boldsymbol{\tau}=\sum_{i} \boldsymbol{J}_{c, i}^{T} \cdot \boldsymbol{F}_{i},
$$

where $\boldsymbol{J}_{c, i}^{T}$ and $\boldsymbol{F}_{i}$ are the contact Jacobian and the contact force of the $i$-th contact, respectively. Contact forces are computed according to the contact force model described in the folowing section and they are reflected to the user through:

$$
\boldsymbol{F}_{e n v, h}=\boldsymbol{J}_{h}^{\dagger} \sum_{i} \boldsymbol{J}_{c, i}^{T} \boldsymbol{F}_{i},
$$

where $\boldsymbol{J}_{h}^{\dagger}$ is the dynamically consistent inverse of the proxy Jacobian at the hand [12].

\subsection{Local contact model}

Contact stability and the perception of rigidity are improved in the local model by computing contact forces using a technique similar to that proposed in [16]. This technique includes a collision impulse upon contact and an interaction force during contact. A shown in Figure 2, each local contact can be in one of three possible contact states: no contact, colliding contact, or continuous (sticking or sliding) contact.

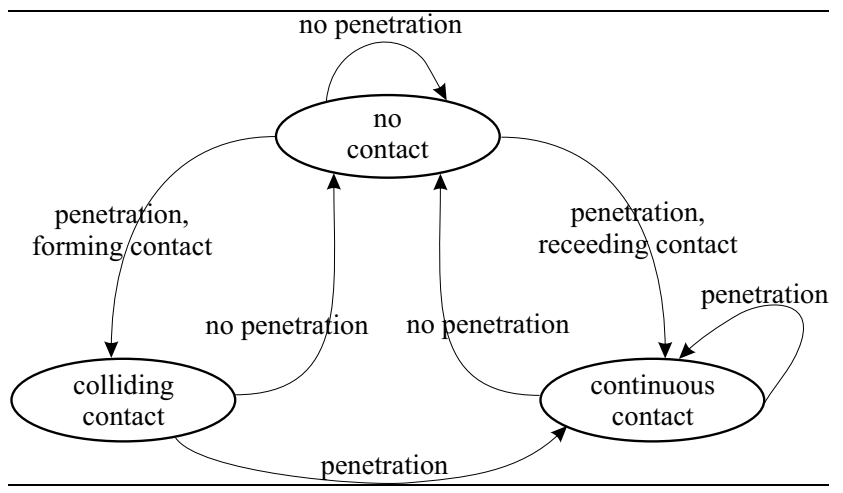

Figure 2. Local contact states.

While state transitions occur based on local contact information, collision resolution must be computed globally. This condition is imposed by the fact that multiple collisions may occur during a single haptic step. However, existing collision resolution schemes model only one rigid body impact and they use heuristics to sequence collisions detected simultanously.

In the proposed local model, post-impact contact velocities are determined at each contact individually based on 
Poisson's restitution hypothesis. Nevertheless, they may impose inconsistent requirements on the post-impact configuration space velocities, i.e., the system:

$$
\left(\begin{array}{c}
\boldsymbol{v}_{c, 1}^{+} \\
\vdots \\
\boldsymbol{v}_{c, n}^{+}
\end{array}\right)=\left[\begin{array}{c}
\boldsymbol{J}_{c, 1} \\
\vdots \\
\boldsymbol{J}_{c, n}
\end{array}\right] \dot{\boldsymbol{q}}^{+}=\boldsymbol{J}_{c} \dot{\boldsymbol{q}}^{+} .
$$

may be inconsistent. In Equation (4), $v_{c, i}^{+}$is the post-impact velocity of the $i$-th contact computed using Poisson's restitution hypothesis and $\dot{\boldsymbol{q}}^{+}$is the post-impact configuration space velocity. The inconsistency is resolved by computing the least squares solution of Equation (4):

$$
\dot{\boldsymbol{q}}^{+}=\left(\boldsymbol{J}_{c}^{T} \cdot \boldsymbol{J}_{c}\right)^{-1} \cdot \boldsymbol{J}_{c}^{T}\left(\begin{array}{c}
\boldsymbol{v}_{c, 1}^{+} \\
\vdots \\
\boldsymbol{v}_{c, n}^{+}
\end{array}\right)
$$

The computed change in configuration space velocities results in a change in the configuration space momentum of the proxy $\boldsymbol{P}_{c s}$ equal to:

$$
\boldsymbol{P}_{c s}=\boldsymbol{D}\left(\dot{\boldsymbol{q}}^{+}-\dot{\boldsymbol{q}}^{-}\right)
$$

where $\dot{\boldsymbol{q}}^{-}$is the configuration space velocity vector before the collisions. The force applied to the user's hand due to the simulataneous collisions can then be computed by transferring the desired configuration space change of momentum at the hand and assuming that it is due to a constant force acting over one haptic step:

$$
\boldsymbol{F}_{e n v, h}=\frac{\boldsymbol{J}_{h}^{\dagger} \boldsymbol{P}_{c s}}{\Delta t},
$$

where $\Delta t$ is the haptic time step.

During continuous contact, the interaction force at the $i$ th contact has a component normal to the contact, modeling stiffness and computed by:

$$
\boldsymbol{F}_{c, i}=\left(K_{c} \boldsymbol{n}_{c, i}^{T}\left(\boldsymbol{c}_{i, e n v}-\boldsymbol{c}_{i}\right)-B_{c} \boldsymbol{n}_{c, i}^{T}\left(\boldsymbol{J}_{c, i} \dot{\boldsymbol{q}}\right)\right) \boldsymbol{n}_{c, i}
$$

and a component tangential to the contact, modeling friction and computed using a simplified Coulomb model. In Equation (8), $\boldsymbol{c}_{i, e n v}$ and $\boldsymbol{n}_{c, i}$ are the constraint position and outward normal received from the virtual environment simulation, $\boldsymbol{c}_{i}$ is the position of the contact on the proxy, and $K_{c}$ and $B_{c}$ are the contact stiffness and damping.

\section{System implementation}

The local model of interaction described in Section 3 has been used to interface a planar twin pantograph haptic device [17] to a virtual environment using the two processor hardware architecture described in [10]. In this architecture, the virtual environment is simulated at about $30-60 \mathrm{~Hz}$ on a 450MHz Pentium III PC running Windows 2000 and it updates the local model infrequently, whenever it has completed a simulation step. The local model is computed at $500 \mathrm{~Hz}$ on the haptic server, a 450MHz Pentium III PC running VxWorks. The haptic server also computes the device control and coordinates force and position information between the local model and the device using a four channel teleoperation controller optimized for transparency [17].

\section{Experiments}

The performance of the proposed local model of interaction is evaluated using the virtual environment depicted in Figure 3. The environment consists of an enclosure of rigis walls (light color), a moving virtual object (dark color), and a grounded articulated structure with three links (last link is shown in light color). The dark region around the virtual articulated structure represents the neighborhood of the virtual tool when the user holds one of the links of this structure. The geometry of this region is used by the local model for contact force computation.

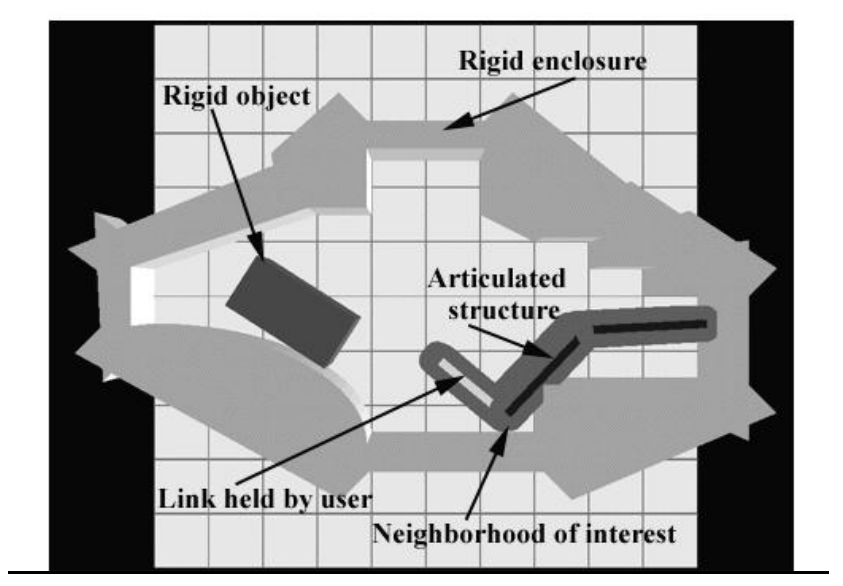

Figure 3. Testbed virtual environment.

Two experiments are performed. The first experiment investigates the stability of contact for two different local contact models: (i) a model comprising penalty-based forces alone and (ii) a model comprising penalty-based forces during continuous contact and impulses upon contact. In the experiment, the user's hand is replaced by a constant force of $2 N$ applied onto a virtual rectangular rigid object. This force drives the object into a virtual wall with stiffness $K_{\text {contact }}=15000 \mathrm{~N} / \mathrm{m}$ and damping $B_{\text {contact }}=20 \mathrm{Ns} / \mathrm{m}$. The results for the two contact models, shown in Figures 4 and 5, demonstrate that collision 
impulses improve the stability of contact in the proposed local model.
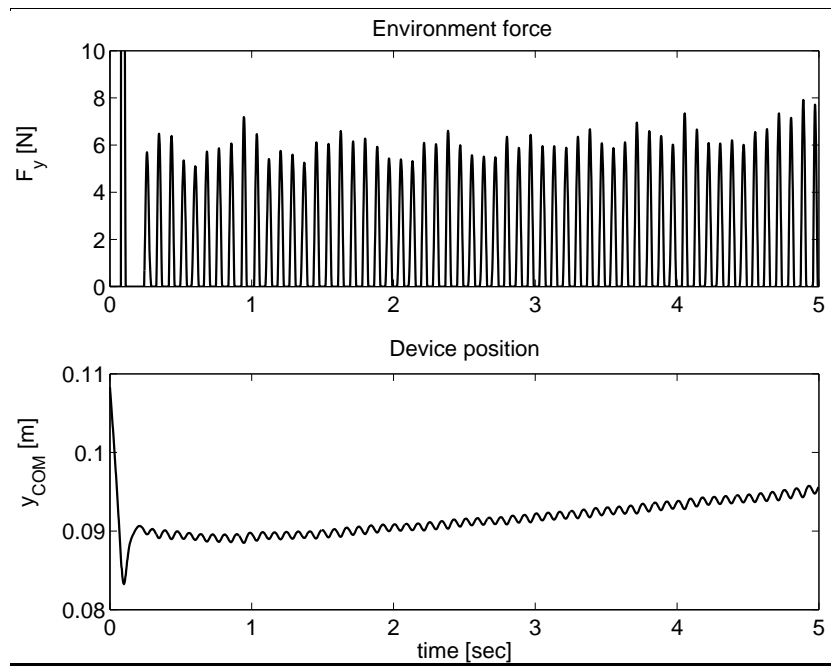

Figure 4. Contact force and device position when an object is pushed into a virtual wall and no colliding contacts exist in the local model.

The second experiment investigates the ability of the configuration space proxy dynamics to impose constraints due to the topology of the virtual tool on the user. The user manipulates the articulated structure shown in the testbed virtual environment by holding it from the third link (light color in Figure 3). The errors between the master and proxy position and the contact forces are plotted in Figures 6 and 7. The device follows the proxy closely, thus demonstrating that the local dynamics can successfully enforce the topology of the structure on the user's hand.

\section{Conclusions}

A local model of interaction developed for haptic rendering of rigid body motion has been extended to the rigid body manipulation of articulated objects. In the proposed local model, motion constraints due to proxy topology are rendered through its mass matrix and motion constraints due to other virtual objects are rendered through contacts. Furthermore, the local contact model is extended to include a collision state, rendered to the user through collision impulses.

The main limitations of the technique are the degradation of performance during swift user motions and the computational expense of local dynamics for large articulated structures. Future work will investigate techniques for improving local geometry based on the prediction of user motion and techniques for reducing the computational burden of local
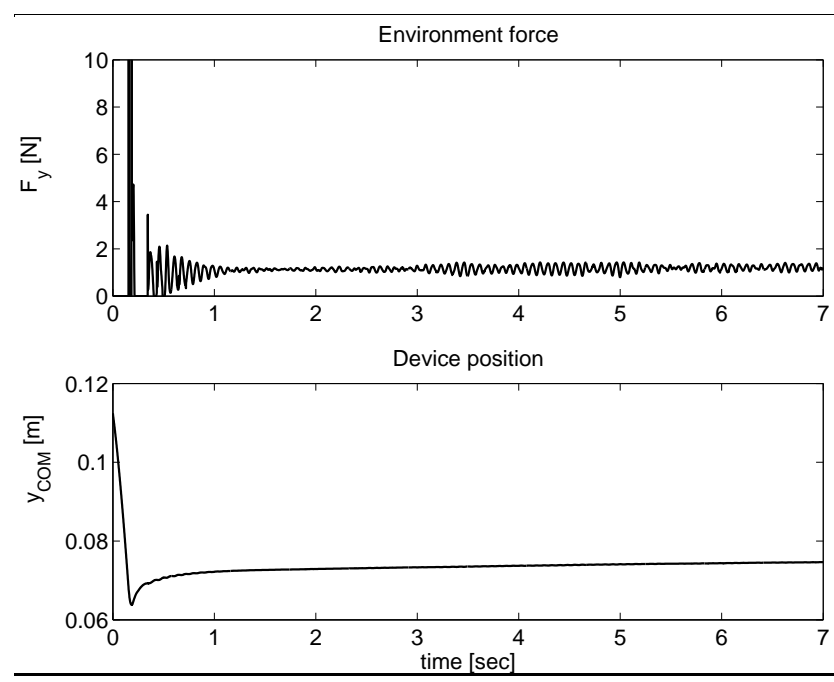

Figure 5. Contact force and device position when an object is pushed into a virtual wall and colliding contacts exist in the local model.

dynamics based on updating it from the virtual environment simulation.

\section{References}

[1] Y. Adachi, T. Kumano, and K. Ogino. Intermediate Representation for Stiff Virtual Objects. In Proceedings of the IEEE Virtual Reality Annual International Symposium, pages 203-210, Research Triangle Park, NC, 1995.

[2] R. Adams and B. Hannaford. Stable Haptic Interaction with Virtual Environments. IEEE Transactions on Robotics and Automation, 15(3):465-474, June 1999.

[3] C. Basdogan, C.-H. Ho, and M. A. Srinivasan. Virtual Environments for Medical Training: Graphical and Haptic Simulation of Laparoscopic Common Bile Duct Exploration. The IEEE/ASME Transactions on Mechatronics, 6(3):269-285, September 2001.

[4] B. Baxter, V. Scheib, M. Lin, and D. Manocha. DAB: Interactive Haptic Painting with 3D Virtual Brushes. In Proceedings of the ACM SIGGRAPH, pages 461-468, University of North Carolina, Chapel Hill, 2001.

[5] J. Berkelman. Tool-Based Haptic Interaction with Dynamic Physical Simulations using Lorentz Magnetic Levitation. PhD thesis, Carnegie Mellon University, 1999.

[6] J. Brown and J. Colgate. Passive Implementation of Multibody Simulations for Haptic Display. In Proceedings of the 1997 ASME International Mechanical Engineering Congress and Exhibition, pages 85-92, Dallas, Texas, 1997.

[7] B. Chang and J. Colgate. Real-Time Impulse-Based Simulation of Rigid Body Systems for Haptic Display. In Proceedings of the 1997 ASME International Mechanical Engineering Congress and Exhibition, pages 1-8, Dallas, Texas, 1997. 

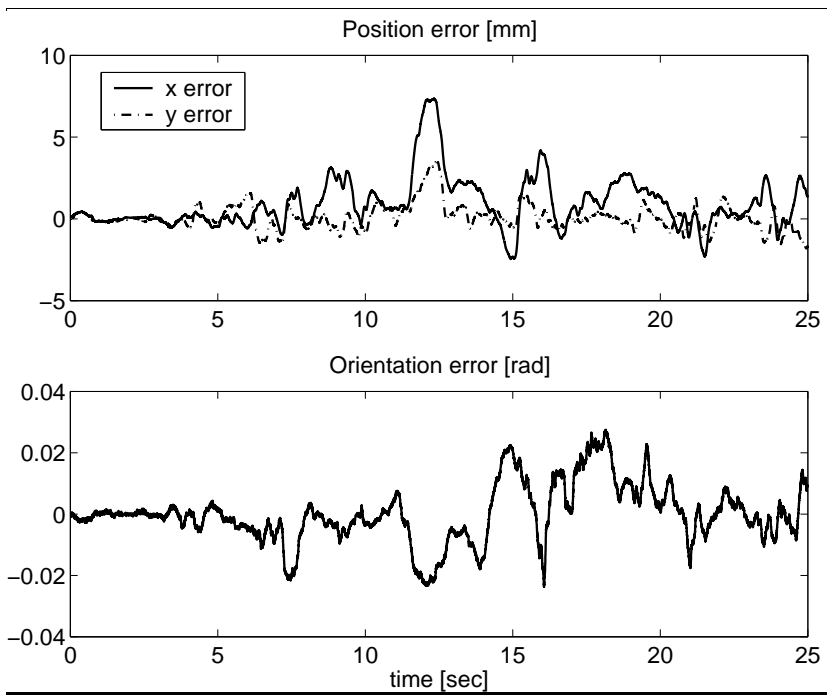

Figure 6. Positional errors between the master and the proxy during the manipulation of an articulated structure.

[8] J. Colgate, M. Stanley, and J. Brown. Issues in the Haptic Display of Tool Use. In Proceedings of the IEEE/RSJ International Conference on Intelligent Robots and Systems, pages 140-145, Pittsburgh, 1995.

[9] D. Constantinescu, I. Chau, L. Filipozzi, S. DiMaio, and S. Salcudean. Haptic Rendering of Planar Rigid-Body Motion using a Redundant Parallel Mechanism. In Proceedings of the IEEE International Conference on Robotics and Automation, pages 2440-2445, Berkeley, Ca, 2000.

[10] D. Constantinescu, S. Salcudean, and E. Croft. Local interaction models for haptic rendering of rigid environments. In 2nd IFAC Conference on Mechatronic Systems, pages 553558, Berkeley, Ca, 2002.

[11] A. Gregory, A. Mascarenhas, S. Ehmann, M. Lin, and D. Manocha. Six Degree-of-Freedom Haptic Display of Polygonal Models. In Proceedings Visualization 2000, pages 139-146, 2000.

[12] O. Khatib. Inertial Properties in Robotic Manipulation: An Object-Level Framework. The International Journal of Robotics Research, 13(1):19-36, 1995.

[13] W. Mark, S. Randolph, M. Finch, J. Van Verth, and R. Taylor II. Adding Force Feedback to Graphics Systems: Issues and Solutions. In Haptic Virtual Reality for Blind Computer Users, Assistive Technologies, pages 92-99, 1998.

[14] D. Ruspini and O. Khatib. Dynamic Models for Haptic Rendering Systems. In Advances in Robot Kinematics: ARK98, pages 523-532, Strob1/Salzburg, Austria, 1998.

[15] D. Ruspini and O. Khatib. Collision/Contact Models for Dynamic Simulation and Haptic Interaction. In Proceedings of the 9th International Symposium on Robotics Research, pages 185-195, Snowbird, Utah, 1999.

[16] S. Salcudean and T. Vlaar. On the Emulation of Stiff Walls and Static Friction with a Magnetically Levitated Input/Output Device. In ASME Haptic Interfaces for Virtual

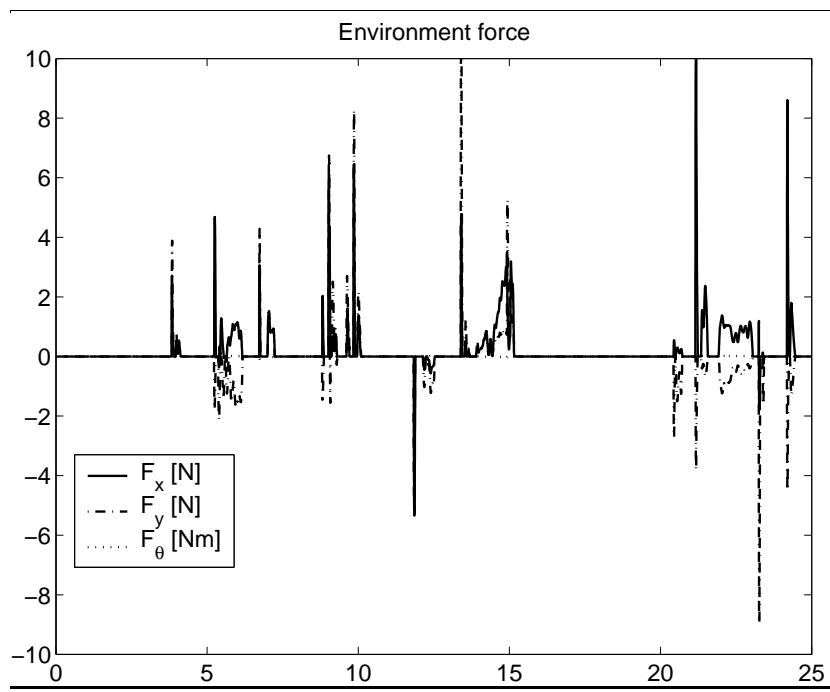

Figure 7. Contact forces during the manipulation of an articulated structure.

Environment and Teleoperator Systems. Dynamics Systems and Control, volume 1, pages 303-309, Chicago, Illinois, 1994.

[17] M. Sirouspour, S. DiMaio, S. Salcudean, P. Abolmaesumi, and C. Jones. Haptic Interface Control - Design Issues and Experiments with a Planar Device. In Proceedings of the IEEE International Conference on Robotics and Automation, pages 789-794, San Francisco, Ca, 2000.

[18] S. Vedula and D. Baraff. Force feedback in interactive dynamic simulation. In Proceedings of the First PHANTOM Users Group Workshop, pages 54-57, 1996.

[19] C. Zilles and J. Salisbury. A Constraint-based God Object Method for Haptic Display. In ASME Haptic Interfaces for Virtual Environment and Teleoperator Systems, Dynamic Systems and Control, pages 146-150, Chicago, IL, 1994. 